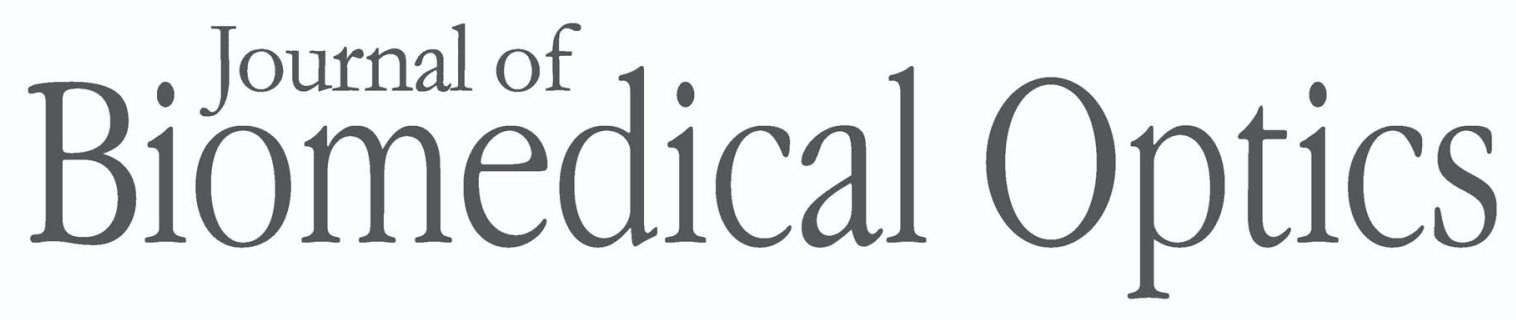

\title{
Effects of motion on optical properties in the spatial frequency domain
}

John Quan Nguyen

Rolf B. Saager

David J. Cuccia

Kristen M. Kelly

James Jakowatz

David Hsiang

Anthony J. Durkin 


\title{
Effects of motion on optical properties in the spatial frequency domain
}

\author{
John Quan Nguyen, ${ }^{a}$ Rolf B. Saager, ${ }^{a}$ David J. Cuccia, ${ }^{b}$ Kristen M. Kelly, ${ }^{\mathrm{c}} J a m e s ~ J a k o w a t z,{ }^{d}$ David Hsiang, ${ }^{\mathrm{d}}$ \\ and Anthony J. Durkin ${ }^{\mathrm{a}}$ \\ a Beckman Laser Institute and Medical Clinic, 1002 Health Sciences Road, Irvine, California 92617-3010 \\ ${ }^{b}$ Modulated Imaging Inc., 1002 Health Sciences Road, Irvine, California 92617-3010 \\ c University of California Irvine, Department of Dermatology, Medical Sciences C-340, Irvine, California 92697-2400 \\ dUniversity of California Irvine, Department of Surgery, 333 City Boulevard West, Suite 700, Orange, California 92868
}

\begin{abstract}
Spatial frequency domain imaging (SFDI) is a noncontact and wide-field optical imaging technology currently being used to study the optical properties and chromophore concentrations of in vivo skin including skin lesions of various types. Part of the challenge of developing a clinically deployable SFDI system is related to the development of effective motion compensation strategies, which in turn, is critical for recording high fidelity optical properties. Here we present a two-part strategy for SFDI motion correction. After verifying the effectiveness of the motion correction algorithm on tissue-simulating phantoms, a set of skin-imaging data was collected in order to test the performance of the correction technique under real clinical conditions. Optical properties were obtained with and without the use of the motion correction technique. The results indicate that the algorithm presented here can be used to render optical properties in moving skin surfaces with fidelities within $1.5 \%$ of an ideal stationary case and with up to $92.63 \%$ less variance. Systematic characterization of the impact of motion variables on clinical SFDI measurements reveals that until SFDI instrumentation is developed to the point of instantaneous imaging, motion compensation is necessary for the accurate localization and quantification of heterogeneities in a clinical setting. $\bigcirc 2011$ Society of Photo-Optical Instrumentation Engineers (SPIE). [DOI: 10.1117/1.3662454]
\end{abstract}

Keywords: spatial frequency domain imaging; modulated imaging; motion correction and compensation.

Paper 11437PR received Aug. 12, 2011; revised manuscript received Oct. 21, 2011; accepted for publication Oct. 25, 2011; published online Dec. 14, 2011.

\section{Introduction}

Spatial frequency domain imaging (SFDI) is a noncontact and wide-field imaging technology currently being developed at the Beckman Laser Institute in Irvine, California. With an interrogation depth of up to $1 \mathrm{~cm}$ below the skin-surface, SFDI is capable of measuring the concentrations of various biological chromophores including hemoglobin (total, oxy-, and deoxyforms), lipid, water, melanin, and tissue oxygen saturation. ${ }^{1}$ The ability to quantitatively characterize the in vivo properties of skin tissue over a wide field of view (FOV) makes SFDI a potentially useful tool in various clinical applications such as thick tissue fluorescence imaging, ${ }^{2}$ vascular occlusion detection, ${ }^{3}$ and monitoring reconstructive tissue status. ${ }^{4}$ However, in the course of developing a clinically deployable SFDI system capable of obtaining high-fidelity optical properties, a compensation strategy for undesired motion in the region of interest must first be addressed. This is particularly true for applications such as the imaging of cutaneous melanoma and benign pigmented lesions. Because of the time and magnification required to effectively image these millimeter-scale targets, ${ }^{5}$ even the slightest amount of patient movement can lead to inaccurate measurements.

In this paper, we demonstrate the need for developing a strategy to correct for the effects of motion on optical properties determined using SFDI. We then develop an algorithm to cor-

Address all correspondence to: John Quan Nguyen, University of California, Irvine, Beckman Laser Institute, 1002 Health Sciences Rd., Irvine, California 92617-3010; Tel: 949-824-3284; E-mail: nguyenjq@gmail.com. rect for these effects and apply it to phantoms and to clinical skin data. Using the approach presented here, we are able to show that the optical properties obtained from moving tissue can be determined with fidelity similar to those determined from stationary tissue.

\subsection{SFDI}

SFDI combines "structured illumination" projections with a camera-based imaging system in order to quantify subsurface absorption $\left(\mu_{a}\right)$ and reduced-scattering $\left(\mu_{s}^{\prime}\right)$ coefficients on a pixel-by-pixel basis. ${ }^{1}$ Deduced optical properties may be diagnostically useful, or can be subsequently further reduced to chromophore concentrations via Beer's law. The SFDI platform consists of three basic components: a light source, a spatial light modulator, and a CCD camera for the detection of remitted light. ${ }^{1}$ During skin imaging, a spatially-modulated illumination scheme with various spatial frequencies is projected over a large area of tissue. The remitted diffuse light is detected via a CCD camera and then demodulated in order to extract the structured ac component of the diffuse reflectance at multiple spatial frequencies. ${ }^{1}$ Due to the differential sensitivity of the diffuse reflectance to absorption and scattering at specific spatial frequencies, these two coefficients can then be reconstructed using algorithms developed in the spatial frequency domain. ${ }^{1}$ By repeating this process through different wavelengths, quantitative tissue chromophore concentrations can be generated.

1083-3668/2011/16(12)/126009/9/\$25.00 @ 2011 SPIE 


\subsection{SFDI Optical Property Determination}

Diffuse light-transport in turbid media is a complex phenomenon governed by multiple absorption and scattering events, both of which can be characterized by absorption coefficients, $\mu_{a}$, and reduced-scattering coefficients, $\mu_{s}^{\prime}{ }^{6}{ }^{6}$ These fundamental optical properties of tissue can provide essential information for many therapeutic and diagnostic applications. ${ }^{2-4}$

Optical properties can be deduced in the spatial frequency domain using the diffusion approximation to the Boltzmann transport equation where the diffuse reflectance is a function of the ratio between the effective attenuation coefficient, $\mu_{\text {eff }}^{\prime}$, and the transport coefficient, $\mu_{\mathrm{tr}}$, where $\mu_{\text {eff }}^{\prime}=\left(3 \mu_{a} \mu_{\mathrm{tr}}+k^{2}\right)^{1 / 2}, \mu_{\mathrm{tr}}$ $=\left(\mu_{a}+\mu_{s}{ }^{\prime}\right)$, and $k$ is the spatial frequency. ${ }^{7}$ As shown by Cuccia et al., the spatial frequency, $k$, is directly related to the contribution of both the absorption and reduced-scattering coefficients to the diffuse reflectance. At lower spatial frequencies close to 0 , the contribution of absorption is predominant and gradually decreases as the spatial frequency increases. ${ }^{1}$ By utilizing this relationship across two or more spatial frequencies, the wavelength-dependent absorption and reduced-scattering coefficients can be solved analytically through a least-squares fitting method.

However, because diffusion approximation is only valid when $\mu_{s}^{\prime} \gg \mu_{a}$, a transport-based forward-model such as a Monte Carlo simulation may be preferable in order to account for a larger range of absorption and reduced-scattering coefficients. ${ }^{1}$ Due to the computationally intensive nature of this method, a rapid two-frequency lookup-table approach was generated from the "white" Monte Carlo simulation of a collimated point source illumination in a purely scattering medium. ${ }^{1,8,9}$ Lookup tables correlate modeled two-spatial frequency (ac and dc) diffuse reflectance to a population of absorption and reduced-scattering coefficient combinations. Compared to the computationally intensive full diffusion model or Monte Carlo simulation, using a lookup table can significantly decrease computation time while maintaining a deviation of less than $10 \%$ from expected reducedscattering values and less than $15 \%$ from expected absorption values. ${ }^{1}$ For example, while a Monte Carlo simulation may take $2 \mathrm{~h}$ in order to compute solutions, the lookup table-based process may take only 6 min running on a $1.86 \mathrm{Ghz}$ dual-core desktop PC with $3 \mathrm{~GB}$ of RAM. In addition, the lookup table approach does not require an initial guess of optical properties, which is especially convenient when extracting optical properties over a wide range of values. ${ }^{1}$

Once optical properties have been computed, chromophore concentrations can be obtained using Beer's Law. Specifically, after obtaining the pixel-by-pixel absorption and reducedscattering coefficients at each wavelength, a basis set of molar extinction coefficients can be fit to the recovered absorption coefficients. Typically, in the 650 to $1000 \mathrm{~nm}$ near-infrared window, these include oxy- and deoxy-hemoglobin, water, and melanin. ${ }^{1}$ Extraction of chromophore concentrations from motion corrected SFDI data acquired will be the focus of future work and will not be discussed further here.

\subsection{Need for SFDI Motion Correction}

While SFDI has been a useful tool in various research endeavors, ${ }^{2-4}$ there is still the important challenge of developing a clinically deployable SFDI system. Part of this challenge, and the main topic of this paper, is related to the development of a strategy for effective compensation of subject motion, which in turn is critical for recording high fidelity optical properties. Depending on the hardware and autoexposure settings, a typical clinical data collection using the SFDI system described above can require the integration of signal for several seconds for each desired combination of wavelengths, spatial frequencies, and phases. During this time, the region-of-interest (ROI) will be displaced slightly by patient movement. The longer an SFDI measurement session takes to acquire data, the more likely the ROI will deviate from its initial position and shift the phase relations of the spatially-modulated projections in the lateral direction of the sinusoid. This, in turn, suggests that SFDI motioncorrection will consist of a two-part procedure:

1. Motion tracking and correction: A method for repositioning and aligning each collected image in order to maintain a consistent ROI for effective image processing.

2. Demodulation correction: A method for demodulation that takes motion-induced arbitrary-phase shifts into consideration for the purpose of generating high-fidelity absorption and reduced-scattering maps.

In order to reposition and align the imaging ROI, a Canny edge detection algorithm is used for the purpose of detecting and tracking the position of a skin-surface fiduciary-marker. Motioninduced phase-shifts are subsequently sampled before the entire image-set is processed by a modified demodulation equation.

\section{Materials and Methods}

\subsection{Instrumentation and Data Acquisition Settings}

In terms of specific instrumentation used in this investigation, the SFDI apparatus consists of a $250 \mathrm{~W}$ tungsten lamp connected to a Newport Corporation (Irvine, California) power source. ${ }^{7}$ This light is used to illuminate the spatially modulated projections created by the $1,024 \times 768$ pixels DLP Developer's Kit digital micromirror device (DMD) (Texas Instruments, Dallas, Texas). Sinusoidal intensity patterns for specified spatial frequencies are generated by a computer and sequentially projected via the DMD at 3 phases: 0,120 , and $240 \mathrm{deg}$. Diffusely reflected light images are acquired using a Nuance Multispectral Imaging System (CRi, Inc., Woburn, Massachusetts) consisting of a liquid-crystal tunable filter capable of passing discrete $10 \mathrm{~nm}$ bandwidth wavelengths between 650 and $1050 \mathrm{~nm}$ into a 1040 $\times 1392$ pixel front-illuminated CCD camera. Specular diffuse skin reflectance is rejected through the incorporation of crosslinear polarizers into the optics of the camera and projection system. The measurement images were saved as binary files for post-acquisition processing. The diffuse reflectance images are calibrated for system response by using a $96 \mathrm{~mm} \times 96 \mathrm{~mm}$ $\times 10 \mathrm{~mm}$ tissue simulating phantom with known optical properties $\left(\mu_{a}=0.0188 \mathrm{~mm}^{-1}\right.$ and $\mu_{s}^{\prime}=1.098 \mathrm{~mm}^{-1}$ at $\left.650 \mathrm{~nm}\right)$. The phantoms were created using silicon-based polydimethylsiloxane with homogeneously distributed India ink as an absorber and $\mathrm{TiO}_{2}$ as a scattering agent. ${ }^{10}$ Spectral absorption and reduced-scattering coefficients of the calibration phantoms were verified using two-distance frequency domain photon migration 


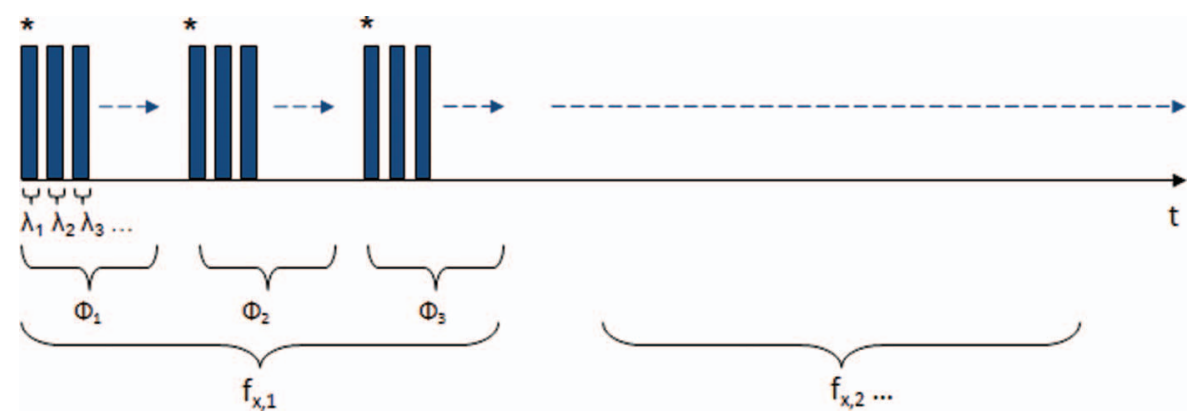

Fig. 1 Diagram illustrating the SFDI acquisition process with respect to time, $t$. Each block represents an image acquired at a specific wavelength, $\lambda$. After the first set of specified wavelengths has been imaged, the same wavelengths are then acquired with the following phase shift, $\Phi$. After all phase shifts are complete, another set of wavelengths and phase shifts are then completed at the next specified spatial frequency until all required measurements are complete. Three phase-shifted images, ${ }^{*}$, are required to generate the demodulated reflectance images for each specified wavelength and frequency combination.

(FDPM) measurement, which is an inherently self-calibrating measurement. ${ }^{11}$

While the FOV for the SFDI system is highly scalable, for the purpose of this study, we maintained a FOV with a $70 \mathrm{~mm}$ $\times 95 \mathrm{~mm}$ dimension. Data acquisition times are largely dependent on the CCD exposure settings for each recorded image. While acquisition times could have been reduced (though not fully eliminated) by imaging fewer wavelengths and frequencies, in order to ensure a comprehensive data-set capable of being reprocessed under wide range of settings for future studies, the following parameters were used for this study: SFDI measurements were acquired at five spatial frequencies equally spaced between 0 and $0.25 \mathrm{~mm}^{-1}$ : the first being the zerofrequency followed by four progressively higher frequencies. Seventeen spectral wavelengths between 650 and $970 \mathrm{~nm}$ were acquired in $20 \mathrm{~nm}$ intervals. Data acquisition times averaged about 2.5 min per measurement comprised of 5 frequencies, 3 phases, 17 wavelengths, and 255 images total.

Figure 1 depicts the acquisition process. Imaging times are dependent on the optical properties of the target at the specified wavelengths, and can vary from $100 \mathrm{~ms}$ up to $3 \mathrm{~s}$. Frequency switching and phase-shifting times are about $20 \mu$ s and are not a significant contribution to the acquisition time. Due to the acquisition times for each imaged wavelength within a phase acquisition as seen in Fig. 1, motion artifacts from seemingly trivial actions such as breathing have the potential of altering the position of the projected phase-shifts in relation to the target. For example, if the total acquisition time for all wavelengths for a given spatial frequency at a given phase shift is about $10 \mathrm{~s}$ and 3 phase shifts are required to generate the demodulated reflectance image, $M_{\mathrm{ac}}$, for a specified spatial frequency and wavelength combination, then the 3 images required for demodulation are inherently separated by about $10 \mathrm{~s}$ in time.

ROI's were manually defined by the user in order to select a large enough region encompassing the lesion and normal skin sample. Optical property determination was achieved by using the two-frequency $\left(0\right.$ and $\left.0.15 \mathrm{~mm}^{-1}\right)$ lookup table for rapid calculation of optical properties generated from Monte Carlo forward predictions as described earlier. In order to reduce the processing time, the lookup table was limited to an absorption range of 0.003 to $0.5 \mathrm{~mm}^{-1}$ and a reduced-scattering range of 0.3 to $6 \mathrm{~mm}^{-1}$. All computations were done in MATLAB using a $1.86 \mathrm{Ghz}$ dual-core desktop PC with $3 \mathrm{~GB}$ of RAM.

\subsection{Motion Tracking and Correction}

The first part of SFDI motion-correction involves motion tracking of the collected images and collating the set so that the ROI in each frame stays consistent throughout post-acquisition data processing. This is accomplished through the use of a fiduciary marker and the Canny edge detection function included in MATLAB. The fiducial is used as a fixed reference point that is easily identifiable by an edge detection operator since it maintains high contrast from tissue at all near-infrared wavelengths imaged with this system. By tracking the frame-to-frame movement of the fiducial, motion information is recorded and used to crop and reposition the image set for consistent data processing as seen in Fig. 2.

The same frame-to-frame motion and ROI cropping information is then applied to a large field-of-view calibration phantom measurement in order to sample (through sine-wave fitting of the summed intensities) the motion-altered phase-shifts, $\Phi_{s}$, for later use in demodulation. This is done once per sample. The use of a well characterized calibration phantom with homogeneous and well-characterized optical properties ensures accurate phase-shift reproductions whereas direct sampling of the

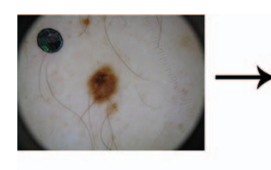

(a)

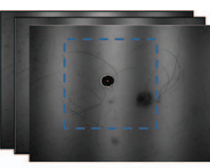

(b)

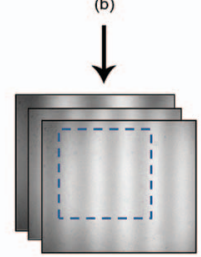

(d)

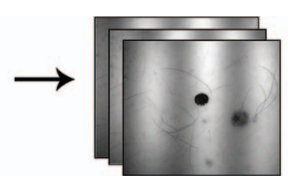

(c)



(e)
Fig. 2 Illustration outlining SFDI motion tracking and correction. (a) A fiduciary marker (upper left) is placed near the pigmented lesion (center) before imaging. (b) During data processing, Canny edge detection is used to mark and track the position of the fiducial. For each individual frame, a ROI is specified with relation to the fiducial's position. (c) The specified ROI's are cropped and collated in order to maintain frame-by-frame consistencies during demodulation. (d) The same ROI is applied to a reference phantom measurement in order to sample ROI specific phase-shifts, (e), for later use in demodulation correction. 
subject's skin would be inaccurate due to the heterogeneities in optical properties present.

Fiducial markers can take the form of just about anything as long as they remain in one place on the subject, are easy to remove after imaging, and are visible at all wavelengths of interest. For this investigation, we used circular shaped reflective stickers (Michael's craft store, Costa Mesa, California). These markers, as opposed to the actual skin-lesion, are used as the motion-tracking reference because skin-lesion surface visibility decreases significantly at longer wavelengths $(>800 \mathrm{~nm})$.

\subsection{Demodulation Correction}

The diffusely-reflected image intensities, $I$, collected by the CCD camera are described as a sum of the illuminated spatially modulated ac and background planar dc components. These can be written as:

$$
\begin{aligned}
& I_{1}(x, y)=M_{\mathrm{ac}} \cos \left(f_{x} x+\Phi_{1}^{\circ}\right)+M_{\mathrm{dc}}, \\
& I_{2}(x, y)=M_{\mathrm{ac}} \cos \left(f_{x} x+\Phi_{2}^{\circ}\right)+M_{\mathrm{dc}}, \\
& I_{3}(x, y)=M_{\mathrm{ac}} \cos \left(f_{x} x+\Phi_{3}^{\circ}\right)+M_{\mathrm{dc}},
\end{aligned}
$$

where $M_{\mathrm{ac}}$ is the magnitude of the spatially-modulated ac component and $M_{\mathrm{dc}}$ is the magnitude of the dc component. $f_{x} x$ and $\phi$ respectfully represent the spatial frequency and phase-shift of the sinusoidal ac pattern. In order to effectively separate the ac and dc components of the diffuse reflectance for use in optical property quantitation, a phase-shifting technique commonly utilized in communication systems is employed. ${ }^{12}$

$$
\left(I_{1}-I_{2}\right)^{2}+\left(I_{2}-I_{3}\right)^{2}+\left(I_{3}-I_{1}\right)^{2} .
$$

The equation above explains why SFDI involves the imaging of three sinusoidal patterns with varying phase-shifts. By subtracting the diffusely-reflected image intensities from one another, the dc component, consisting of average-image noise, digitization offset, ambient lighting, and potential spatial-calibration errors, is removed. ${ }^{1}$ Also, when the phase-shifts are symmetrical at exactly 0,120 , and $240 \mathrm{deg}$, the equation reduces into $9 / 2 * M_{\mathrm{ac}}{ }^{2}$, thus allowing ac extraction to be as simple as squarerooting and multiplying by a constant:

$$
\left.M_{\mathrm{ac}}(x, y)\right|_{f_{x}}=\frac{\sqrt{2}}{3} \sqrt{\left(I_{1}-I_{2}\right)^{2}+\left(I_{2}-I_{3}\right)^{2}+\left(I_{3}-I_{1}\right)^{2}},
$$

while Eq. (3) above works well when dealing with stationary objects, symmetrical 120 deg phase-shifts cannot always be achieved when imaging live tissues in a clinical environment. Considering the small size of many pigmented lesions $(\sim 5 \mathrm{~mm})$ and the amount of camera-zoom required to effectively image the lesion, motion artifacts from seemingly trivial actions such as breathing have the potential of altering the position of the projected phase-shifts in relation to the target which leads to the creation of arbitrary and unintended phase-shift values.

This leads us to the second part of motion-correction in SFDI: modification of the demodulation equation in order to account for motion-related phase-shifts. For arbitrary phase-shifts in Eq. (1), Eq. (2) will reduce into a value consisting of a function, $\kappa(x)$, multiplied by the ac magnitude squared:

$$
\left(I_{1}-I_{2}\right)^{2}+\left(I_{2}-I_{3}\right)^{2}+\left(I_{3}-I_{1}\right)^{2}=\kappa(x) M_{\mathrm{ac}}^{2} .
$$

SFDI motion-correction aims to remove the $\kappa(x)$ function and isolate $M_{\mathrm{ac}}{ }^{2}$ by dividing it by a correction factor, $\kappa_{s}(x)$, based on Eq. (1) and utilizing the sampled phase-shifts, $\Phi_{s}$, described in Sec. 2.2.

$$
\begin{aligned}
\kappa_{s}(x)= & {\left[\cos \left(f_{x} x+\Phi_{1}^{\circ}\right)-\cos \left(f_{x} x+\Phi_{2}^{\circ}\right)\right]^{2}+} \\
& {\left[\cos \left(f_{x} x+\Phi_{2}^{\circ}\right)-\cos \left(f_{x} x+\Phi_{3}^{\circ}\right)\right]^{2}+} \\
& {\left[\cos \left(f_{x} x+\Phi_{3}^{\circ}\right)-\cos \left(f_{x} x+\Phi_{1}^{\circ}\right)\right]^{2} . }
\end{aligned}
$$

When phase-shifts are accurately sampled, $\kappa_{s}(x)=\kappa(x)$ and we are able to isolate $M_{\mathrm{ac}}$ from Eq. (4). This can be rewritten as Eq. (6).

$$
\left.M_{\mathrm{ac}}(x, y)\right|_{f_{x}}=\sqrt{\frac{\left(I_{1}-I_{2}\right)^{2}+\left(I_{2}-I_{3}\right)^{2}+\left(I_{3}-I_{1}\right)^{2}}{\kappa_{s}(x)}} .
$$

The ac and dc values extracted from this method can then be processed using either the Monte Carlo simulation or the twofrequency lookup table for rapid calculation of optical properties as described earlier.

\subsection{Clinical Subjects}

In vivo data was collected under the IRB approved protocol HS No. 2008-6307. Under this protocol, patients with suspicious skin-lesions were consented and imaged before subsequently being diagnosed by a dermatologist.

SFDI data was gathered from 7 subjects, including 1 case study subject. All lesions were clinically benign. Subjects were all middle-aged and light-skinned Caucasian. Data was subsequently analyzed with and without the motion correction scheme described herein.

\section{Results and Analysis}

\subsection{Testing and Validation on Tissue Simulating Phantoms}

In order to assess the validity of the motion-correction technique, moving phantom measurements were conducted followed by measurements in which the phantom was stationary. Artificial tissue-simulating phantoms with superficial lesions located at the surface ( $5 \mathrm{~mm}$ in diameter and $3 \mathrm{~mm}$ deep) were created from silicon-based polydimethylsiloxane with homogeneously distributed India ink as an absorber and $\mathrm{TiO}_{2}$ as a scattering agent. ${ }^{10}$ The absorption and reduced scattering coefficients of the test-lesion was $\mu_{a}=0.299 \mathrm{~mm}^{-1}$ at $650 \mathrm{~nm}$ and $\mu_{s}^{\prime}$ $=1.013 \mathrm{~mm}^{-1}$ at $650 \mathrm{~nm}$, respectively. The absorption and reduced scattering coefficients of the phantom-skin surrounding the lesion was $\mu_{a}=0.0233 \mathrm{~mm}^{-1}$ at $650 \mathrm{~nm}$ and $\mu_{s}^{\prime}=0.728$ $\mathrm{mm}^{-1}$ at $650 \mathrm{~nm}$, respectively. Both were verified using twodistance, multifrequency FDPM measurement, as mentioned in Sec. 2.1.

Stationary phantom measurements were used both as a gold standard reference for the evaluation of the correction method on data collected from a moving phantom and to verify that this correction method does not introduce any additional errors or artifacts when applied to stationary samples. Under ideal, stationary conditions, SFDI measurements would consistently maintain symmetrical 120 deg phase-shifts and the motion-corrected demodulation equation, Eq. (6), would simplify into the noncorrected demodulation formula, Eq. (3). In this case, both 


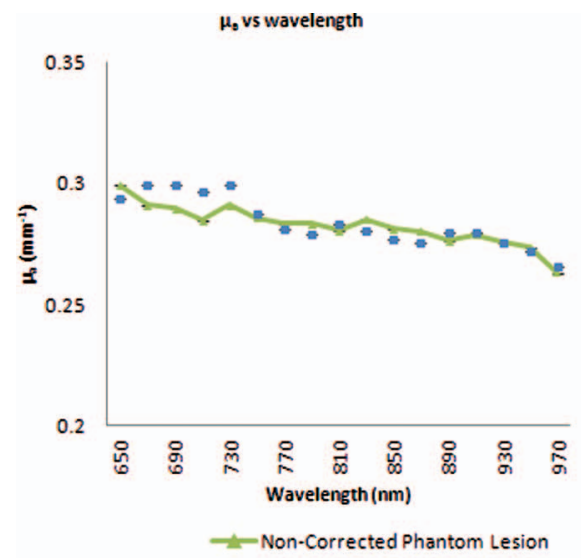

(a)



(b)

Fig. 3 Comparison between noncorrected and motion-corrected SFDI processing on a stationary phantom-lesion measurement. The absorption versus wavelength plots (a) and reduced-scattering versus wavelength plots (b) in both results show no significant difference $(<1.3 \%)$ between noncorrected and motion corrected data, thus implying that the proposed motion-correction technique does not induce artifacts into the data processing.

processing methods should become identical and produce similar results. With motion involved, the phase-shifts and ROI's would no longer be consistent and any noncorrected data would be skewed. Figures 3(a) and 3(b) show the results from a stationary phantom-measurement, and Figs. 4(a) and 4(b) show the results from a moving-phantom measurement. Data points on the plots indicate the ROI pixel averages while error bars indicate the distribution of variances within the same ROI.

In Fig. 3, there are no significant differences $(<1.3 \%$ for any wavelength measured) between the absorption and scattering results when using both the standard and motion correction processing methods. Variance is minuscule in the absorption and scattering plots for both noncorrected and motion-corrected results. The lack of difference in the results implies that the proposed motion-correction technique does not introduce artifacts into the data processing despite the requirement of additional data sampling and processing steps, and suggests that the proposed motion-correction technique is valid for additional clinical studies.
Further validating the technique and illustrating its necessity are the "absorption versus wavelength" [Fig. 4(a)] and "reduced-scattering versus wavelength" [Fig. 4(b)] plots from the moving phantom measurement. In order to simulate arbitrary phase-shifts, the phantom was displaced about $2.5 \mathrm{~mm}$ manually in the lateral direction of the sinusoid at approximately $0.5 \mathrm{~Hz}$ during the entire measurement. This amount and rate of displacement is much higher than would be expected in a clinical setting and serves as an extreme case for motion-correction. Motion-corrected results are nearly identical to those found in Fig. 3, while the noncorrected data exhibits large variances and wide differences between wavelength specific mean values compared to the motion-corrected data. This is most likely attributed to crosstalk between the testlesion's and phantom-skin's contrasting optical properties and can be visually seen in optical property maps as a "blurring" artifact in Fig. 5(a). Reduced-scattering values [Fig. 4(b)] in particular were most affected due to increased sensitivity at higher spatial-frequencies, ${ }^{1}$ and because of the greater relative

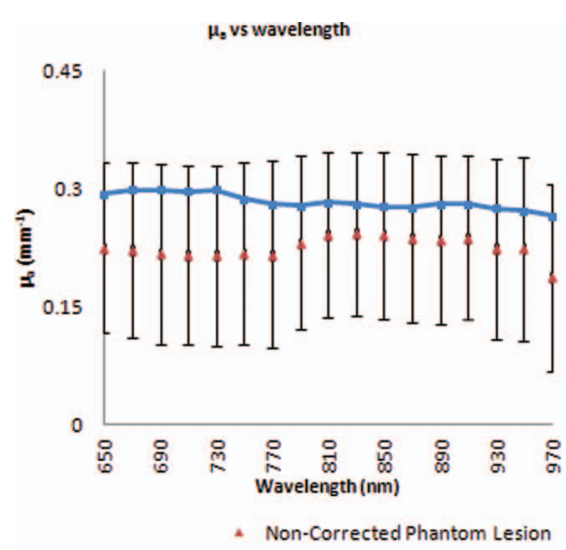

(a)

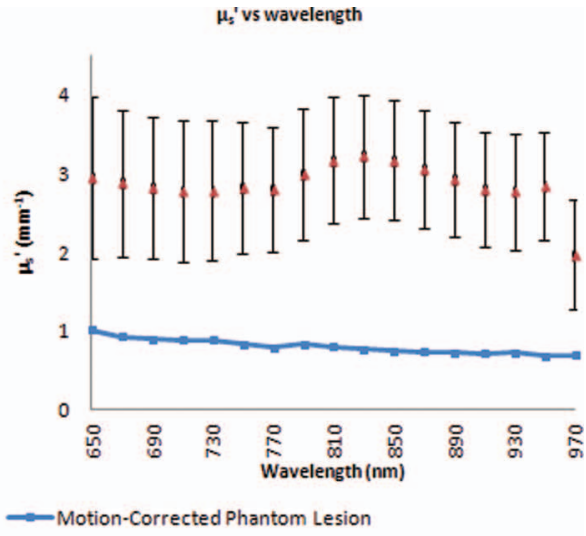

(b)

Fig. 4 Comparison between noncorrected and motion-corrected SFDI processing on a moving phantom-lesion measurement. Both the absorption versus wavelength (a) and reduced-scattering versus wavelength plots (b) exhibit a wide difference between wavelength specific mean values for noncorrected and motion corrected data. Large variances can also be seen in the noncorrected data. 
Nguyen et al.: Effects of motion on optical properties in the spatial frequency domain



(a)

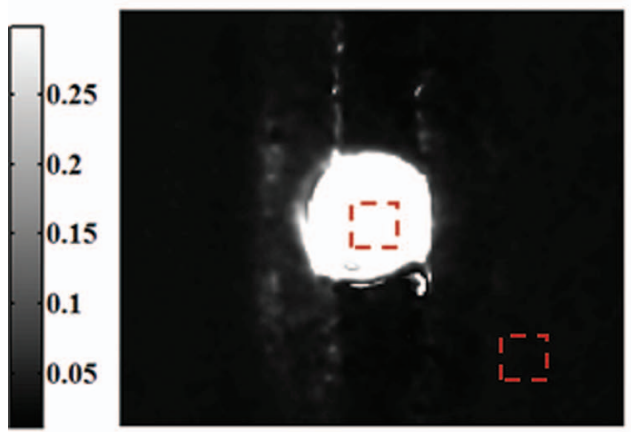

(c)
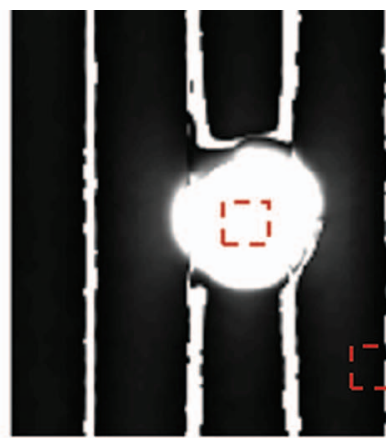

(b)

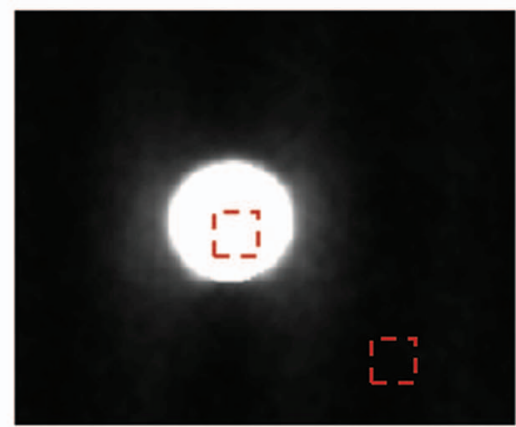

(d)

Fig. 5 Comparison of absorption maps at $650 \mathrm{~nm}$ for a moving test lesion where: (a) no motion correction is applied, (b) images are reregistered to compensate for motion, but the demodulation calculation still assumes 120 deg phase separation [i.e., Eq. (3)] (c) is motion corrected using a consistent ROI and demodulation correction. (d) The absorption map at $650 \mathrm{~nm}$ for an ideal stationary test phantom. Dashed squares indicate areas sampled for extracting data.

phase-shifts produced by motion artifacts at higher spatial frequencies.

Figure 5 also illustrates the consequences of only applying intermediate steps of this correction process. Fig. 5(a) shows the resulting absorption map of the lesion at $650 \mathrm{~nm}$ without applying any motion correction. Here the lesion is blurred laterally. As the lowest spatial frequency holds the greatest contrast with absorption, the overlaid three circular lesions in this image actually represent the motion artifact induced between the three phase images acquired at the spatial frequency, $f_{x}=0 \mathrm{~mm}^{-1}$. Reregistering the images in order to maintain consistent ROI's of the lesion removes this artifact; however, without demodulation correction, introduces significant background noise into the extracted data ["striations" in Fig. 5(b)] due to phase-shift errors. ${ }^{1}$ In order to compensate for all motion-related effects in SFDI, both ROI correction and demodulation correction must be implemented in order to reduce all motion artifacts as seen in Fig. 5(c).

\subsection{Clinical Case Study}

After verifying that the motion-correction technique was comparable to the original noncorrected method, measurements on human subjects were conducted. The goal of these experiments was to verify the algorithm's deduction of optical properties from moving tissue and to determine the adequacy of the overall approach. In presenting and discussing the results of this study, we will first show the results from two case studies followed by a set of clinical measurements.
Both the stationary and moving results presented below were collected from the same subject, a middle-aged Caucasian male with a 5-mm diameter pigmented-lesion located on the upperright portion of their back. In both measurements, the subject was prone on a hospital gurney.

Stationary case study. During this measurement, the subject was instructed to lie as still as possible. Regardless, autonomic actions such as breathing and involuntary twitches were still present. Motion tracking data indicated lesion displacements of up to $0.5 \mathrm{~mm}$ (the lesion itself was about $5-\mathrm{mm}$ wide).

Looking at the absorption versus wavelength and reducedscattering versus wavelength plots for the noncorrected data set [Figs. 6(a) and 6(c)], it is apparent that even the slightest movement can cause a significant increase in optical property variance for the ROI's, especially in the shorter wavelengths (650 to $730 \mathrm{~nm}$ ) where melanin is a primary absorber. It is also clear that motion-correction minimizes the variance in optical properties. For this particular stationary clinical subject, an $83.88 \%$ variance decrease was observed in the absorption versus wavelength plot after applying motion-correction. An $84.29 \%$ variance decrease was observed in the scattering versus wavelength plot after applying motion-correction.

Moving case study. During this measurement, the same subject was instructed to engage in small-talk with the clinician during skin-imaging. The motivation was to generate motion artifacts larger than autonomic functions and represent a more extreme, yet clinically relevant, acquisition setting. Motion 


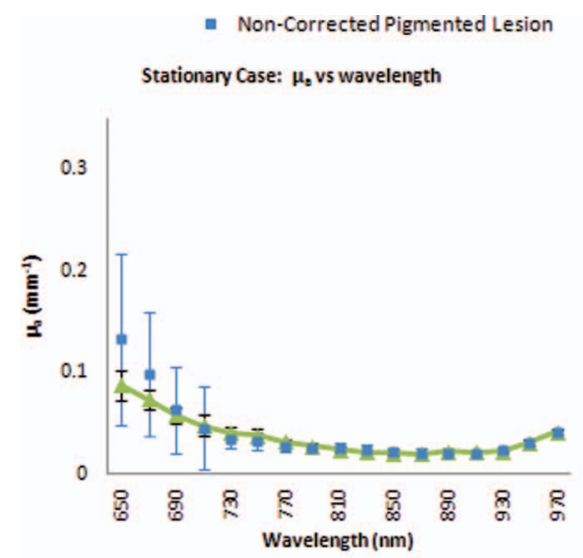

(a)



(c)

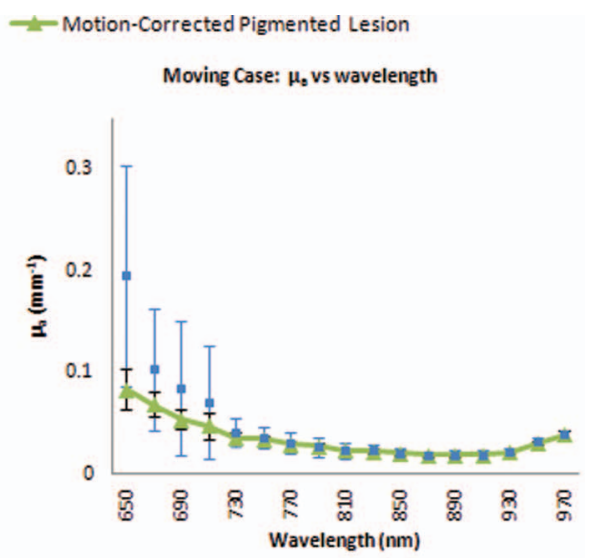

(b)

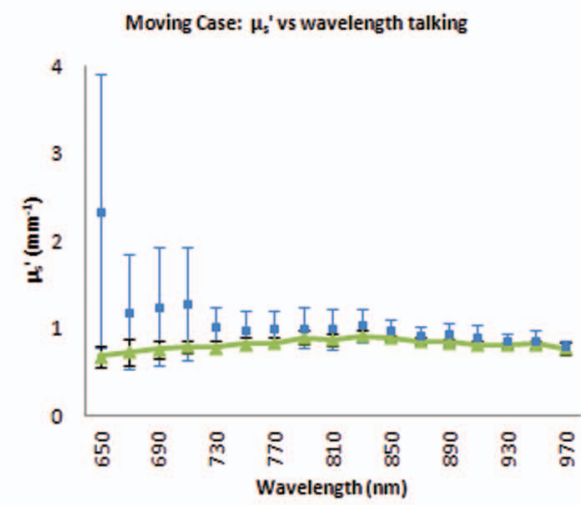

(d)

Fig. 6 Comparison between noncorrected and motion-corrected SFDI processing on the case subject's 5-mm wide skin-lesion. Stationary measurements [(a) and (c)] included lesion displacements of up to $0.5 \mathrm{~mm}$ while moving measurements [(b) and (d)] included lesion displacements of up $7.1 \mathrm{~mm}$. At the shorter wavelengths $(650$ to $710 \mathrm{~nm}$ ), there is a noticeable decrease in variance between the noncorrected and motion-corrected absorption versus wavelength plots [(a) and (b)]. A similar trend can be seen between the noncorrected and motion-corrected reduced-scattering versus wavelength plots [(c) and (d)].

tracking data indicated lesion displacements of up to $7.1 \mathrm{~mm}$ (more than an order-of-magnitude larger than the stationary case described earlier).

From the results illustrated in Fig. 6(b) and 6(d), it can be observed that the amount of optical property variance further increases the more the subject moves. Similar to the stationary measurements, the moving clinical measurements expressed a large amount of variance, especially at the shorter 650 to $730 \mathrm{~nm}$ wavelengths where melanin is a primary absorber. For this case, an $85.58 \%$ variance decrease was observed in the absorption versus wavelength plot after enabling motion-correction. A $92.63 \%$ decrease in variance was observed in the reduced-scattering versus wavelength plot after enabling motion-correction.

It is interesting to note that the motion-corrected optical properties found in the moving measurement [Fig. 6(b) and 6(d)] are nearly identical to those found in the motion-corrected stationary measurements [Fig. 6(a) and 6(c)] with differences of less than $1.5 \%$ for each wavelength. This is a very encouraging result and is another indicator that the motion-correction scheme operates as expected.

Average results from other clinical measurements. The results presented in this section represent the average optical prop- erty values from six other clinical measurements. Unlike the case-study described earlier, these subjects were only imaged once and were only instructed to move as little as possible during the measurements. Lesions varied in locations around the body, but were all imaged on a relatively flat two-dimensional (2D) plane as the depth of focus of the instrument is $\pm 1 \mathrm{~cm}$. Data acquisition and processing methods have been developed to address surface curvatures of a larger $\pm 3 \mathrm{~cm}$ range; ${ }^{13}$ however, these methods were not deployed in this study because the goal was to evaluate the performance of the 2D motion correction technique. The average skin-lesion size was $4.16 \mathrm{~mm}$ in diameter with the smallest lesion having a 3-mm width and the biggest lesion having a 6-mm width. Motion tracking indicated an average fiducial displacement of about $2.08 \mathrm{~mm}$ per measurement, with $0.5 \mathrm{~mm}$ being the smallest displacement and 5 $\mathrm{mm}$ being the largest displacement.

As shown in Fig. 7, the average optical property plots for these six other clinical measurements display very similar trends to those found in the case study depicted in Fig. 6. At the shorter 650 to $730 \mathrm{~nm}$ wavelengths where melanin is a primary absorber, data distribution is much more pronounced and varied in the noncorrected absorption versus wavelength [Fig. 7(a)] and reduced-scattering versus wavelength plots [Fig. 7(c)]. While the large error bars could be solely due to 


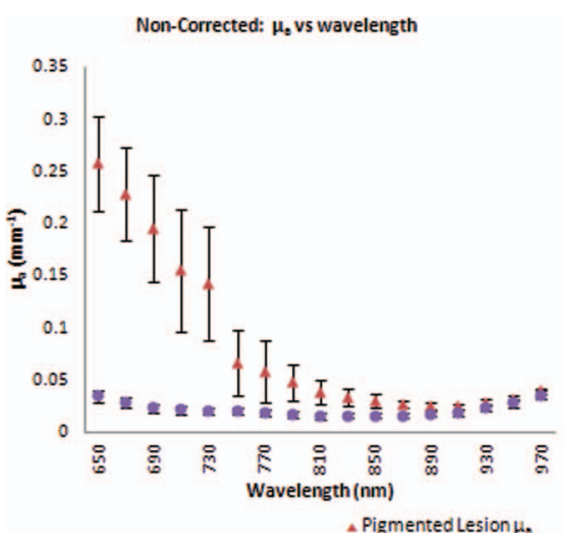

(a)

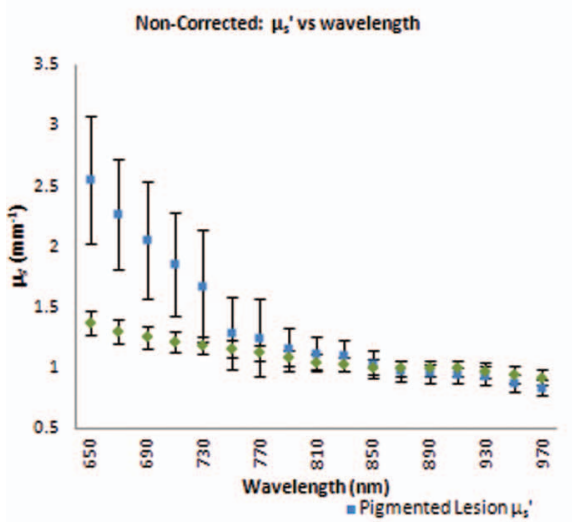

(c)

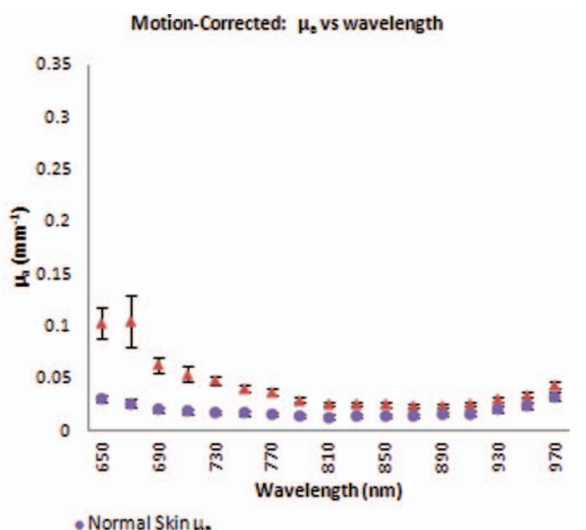

(b)

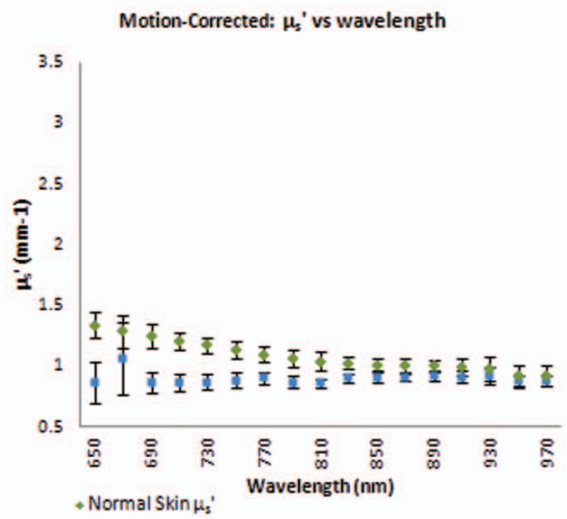

(d)

Fig. 7 Comparison between the average optical property results from six clinical patient measurements using noncorrected [(a) and (c)] and motioncorrected [(b) and (d)] SFDI processing. Similar to the results found in the previous case study (Fig. 6), there is a very noticeable decrease in data variation between the noncorrected (a) and motion-corrected (b) absorption versus wavelength plots at the shorter 650 to $730 \mathrm{~nm}$ wavelengths. The same trend is noticeable between the noncorrected (c) and motion-corrected (d) reduced-scattering versus wavelength plots, however, with a much more significant impact where the noncorrected reduced-scattering versus wavelength plot (c) implies that reduced-scattering values are generally larger in pigmented lesions than compared to normal skin. The motion-corrected reduced-scattering versus wavelength plot (d) suggests otherwise.

interpatient variation in absorption and scattering, had this been the case, the variations would have also been apparent in the motion-corrected plots [Fig. 7(b) and 7(d)]. When comparing the results, it can be seen that the error bars in the motioncorrected plots are significantly smaller than those found in the noncorrected plots.

\section{Discussion}

The results presented here indicate that, if uncompensated, motion can play a large role in skewing SFDI measurement data. This is especially true in the 650 to $730 \mathrm{~nm}$ wavelengths where lesion absorption and reduced-scattering coefficient variability were the largest.Encouragingly, motion-corrected absorption coefficient plots from these experiments are comparable to other studies ${ }^{14,15}$ and are consistent with our understanding of skin chromophore optical properties. Large optical property variances in the shorter wavelengths $(650$ to $730 \mathrm{~nm})$ for the nonmotion corrected data sets are most likely attributed to motionrelated crosstalk between the lesion and normal skin optical property values. Melanin is a primary absorber at these wavelengths and it is generally understood that pigmented lesions are comprised of a relatively large fraction of melanin compared to normal skin. ${ }^{16}$ At longer wavelengths (730 to $970 \mathrm{~nm}$ ), melanin is less absorbing and the optical property differences between pigmented lesions and normal skin becomes less apparent. Because the motion induces arbitrary phase-shifts relative to the fixed position of the ROI, in the absence of motion compensation, both lesion and normal-skin optical properties mix and lead to widely varying optical properties at shorter wavelengths. At longer wavelengths, melanin no longer becomes a large differentiating factor between pigmented lesion and normal skin optical properties, and this helps to explain the smaller error bars at these wavelengths. The slight increase in absorption at $970 \mathrm{~nm}$ for both the normal skin and pigmented lesion is attributed to the water absorption peak at this range. ${ }^{17}$ It is also clear from Fig. 7 that normal-skin presented very consistent optical properties that are comparable to other published studies ${ }^{6}$ regardless of motion compensation, which is reassuring in terms of the performance of SFDI as it applies to skin.

For our measurement paradigm, the largest and most critical type of motion observed was lateral displacement in the direction of the projected sinusoids. Axial or tissue deformation has not contributed significantly to the artifacts present in the data as they have typically remained minimal. Overall, the technique addresses two sources of errors: target displacement and arbitrary phase shifts (both being the result of 2D motion-artifacts in the $x$ and $y$ direction). The technique does not address the effects 
of tissue curvature and profilometry, however, these issues have been addressed in another paper originating from our lab. ${ }^{13}$

\section{Conclusion}

This study demonstrates that motion can play a large role in introducing errors into SFDI measurement results. The more a subject moves during imaging, the larger the variance in optical properties will be if left uncompensated. This is especially true in terms of studying the optical properties of pigmented lesions in a clinical setting, as shown in Fig. 6 and Fig. 7. The smaller ROI that encompasses the lesion compared to the rest of the surrounding normal-skin makes it more difficult to obtain highfidelity in vivo optical property SFDI measurements without the use of motion-correction. In the end however, the ideal scenario would be an SFDI platform in which motion-correction would not even be necessary.

The main reason why motion is currently an issue is related to the acquisition time required to complete a SFDI measurement under the current system configuration. It is important to note that this is not a fundamental limitation of the SFDI technique, but rather a limitation of the current instrumentation. There are several ways to help improve acquisition time on the current system, such as imaging fewer frequencies at fewer wavelengths (effectively improving the acquisition time by a factor of 2 or more) and by improving the light throughput of the instrument. However, when engaging in investigational studies such as this, there is a greater importance placed on acquiring more frequencies and wavelengths in order to determine which combination may best characterize the tissue of interest.

\section{Acknowledgments}

The authors gratefully acknowledge funding provided by the NIH SBIR 1R43RR030696-01A1, the NIH NCRR Biomedical Technology Research Center (LAMMP: 5P-41RR01192), the Military Photomedicine Program, AFOSR Grant No. FA955008-1-0384, and the Beckman Foundation.

\section{References}

1. D. J. Cuccia, F. Bevilacqua, A. J. Durkin, F. R. Ayers, and B. J. Tromberg, "Quantitation and mapping of tissue optical properties using modulated imaging," J. Biomed. Opt. 14, 024012 (2009).

2. A. Mazhar, D. J. Cuccia, S. Gioux, A. J. Durkin, J. V. Frangioni, and B. J. Tromberg, "Structured illumination enhances resolution and contrast in thick tissue fluorescence imaging," J. Biomed. Opt. 15 010506 (2010).

3. M. R. Pharaon, T. Scholz, S. Bogdanoff, D. Cuccia, A. J. Durkin, D. D. Hoy, and G. R. D. Evans, "Early detection of complete vascular occlusion in a pedicle flap model using quantitative spectral imaging," J. Plast. Reconstr Surg. Nurs. 126(6), 1924-1935 (2010).

4. A. Yafi, T. S. Vetter, T. Scholz, S. Patel, R. B. Saager, D. J. Cuccia, G. R. Evans, and A. J. Durkin, "Postoperative quantitative assessment of reconstructive tissue status in cutaneous flap model using spatial frequency domain imaging," J. Plast. Reconstr Surg. Nurs. 127(1), 117-130 (2011).

5. A. Breslow, "Thickness, cross-sectional areas and depth of invasion in the prognosis of cutaneous melanoma," Ann. Surg. 172, 902-908 (1970).

6. S. H. Tseng, P. Bargo, A. Durkin, and N. Kollias, "Chromophore concentrations, absorption and scattering properties of human skin in-vivo," Opt. Express 17, 14599-14617 (2009).

7. F. R. Ayers, D. J. Cuccia, K. M. Kelly, and A. J. Durkin, "Wide-field spatial mapping of in vivo tattoo skin optical properties using modulated imaging," Lasers Surg. Med. 41, 442-453 (2009).

8. A. Kienle and M. S. Patterson, "Determination of the optical properties of turbid media from a single Monte Carlo simulation," Phys. Med. Biol. 41, 2221-2227 (1996).

9. J. Swartling, A. Pifferi, A. M. Enejder, and S. Andersson-Engels, "Accelerated Monte Carlo models to simulate fluorescence spectra from layered tissues," J. Opt. Soc. Am. 20, 714-727 (2003).

10. F. Ayers, A. Grant. D. Kuo, A. J. Durkin, and D. J. Cuccia, "Fabrication and characterization of silicone-based tissue phantoms with tunable optical properties in the visible and near infrared domain," Proc. SPIE 6870, 687007 (2008).

11. R. C. Haskell, L. O. Svaasand, T. T. Tsay, T. C. Feng, M. S. McAdams, and B. J. Tromberg, "Boundary conditions for the diffusion equation in radiative transfer," J. Opt. Soc. Am. 11, 2727-2741 (1994).

12. D. J. Cuccia, F. Bevilacqua, A. J. Durkin, and B. J. Tromberg, "Modulated imaging: quantitative analysis and tomography of turbid media in the spatial-frequency domain," Opt. Lett. 30, 1354-1356 (2005).

13. S. Gioux, A. Mazhar, D. J. Cuccia, A. J. Durkin, B. J. Tromberg, and J. V. Frangioni, "Three-dimensional surface profile intensity correction for spatially modulated imaging," J. Biomed. Opt. 14, 034045 (2009).

14. R. Marchesini, M. Brambilla, C. Clemente, M. Maniezzo, A. E. Sichirollo, A. Testori, D. R. Venturoli, and N. Cascinelli, "In vivo spectrophotometric evaluation of neoplastic and non-neoplastic skin pigmented lesions-I. Reflectance measurements," Photochem. Photobiol. 53, 77-84 (1991).

15. N. Kollias and G. N. Stamatas, "Optical non-invasive approaches to diagnosis of skin diseases," J. Invest. Dermatol. 7, 64-75 (2002).

16. S. L. Jacques and D. J. McAuliffe, "The melanosome: threshold temperature for explosive vaporization and internal absorption coefficient during pulsed laser irradiation," Photochem. Photobiol. 53, 769-775 (1991).

17. F. Bevilacqua, A. J. Berger, A. E. Cerussi, D. Jakubowski, and B. J. Tromberg, "Broadband absorption spectroscopy in turbid media by combined frequency-domain and steady-state methods," Appl. Opt. 39, 6498-6507 (2000). 